Equations of State and scale effect implementation for 1-D Modelling of Performance in Ram Accelerator Thermally Choked Propulsion Mode

\title{
Pascal Bauer
}

Laboratoire PPRIME

CNRS-ENSMA Poitiers, France

E-mail : pascal.bauer@1cd.ensma.fr

\section{Tarek Bengherbia}

Laboratoire PPRIME

CNRS-ENSMA Poitiers, France

E-mail :

tarek.bengherbia@lcd.ensma.fr

\section{Carl Knowlen}

University of Washington

Seattle, WA 98195, WA, USA

E-mail: knowlen@aa.washington.edu

\section{Adam Bruckner}

University of Washington

Seattle, WA 98195, WA, USA

E-mail: bruckner@aa.washington.edu

\section{Yufeng Yao}

University of the West of England,

Department of Engineering Design and Mathematics, Bristol BS16 1QY, UK

E-mail: y.yao@kingston.ac.uk

\section{Marc Giraud}

Exobal Consulting

Saint-Louis la Chaussée, 68300, France

exobal@wanadoo.fr

\begin{abstract}
This paper presents advancement on one-dimensional (1-D) unsteady modelling of a ram accelerator (RAMAC) in the sub-detonative velocity regime by including real-gas equations of state (EoS) in order to account for the compressibility effects of the combustion products. Several equations of state based on generalized empirical and theoretical considerations are incorporated into a 1-D computer code TARAM. The objective of this work is to provide the best available formulations in order to improve the unsteady 1-D model and make the TARAM code a useful tool to predict the performance of the RAMAC in the sub-detonative velocity regime, without having to resort to more complicated 2-D or 3-D computational schemes. The calculations are validated against experimental data from 38-mm and 90-mm-bore facilities and good agreements have been achieved. Yet, the results demonstrate the need for further CFD studies involving the scale effect.
\end{abstract}

Keywords: Detonation, Equation of State, Ram Accelerator, Propulsion, Scale Effects. 


\section{Nomenclature}

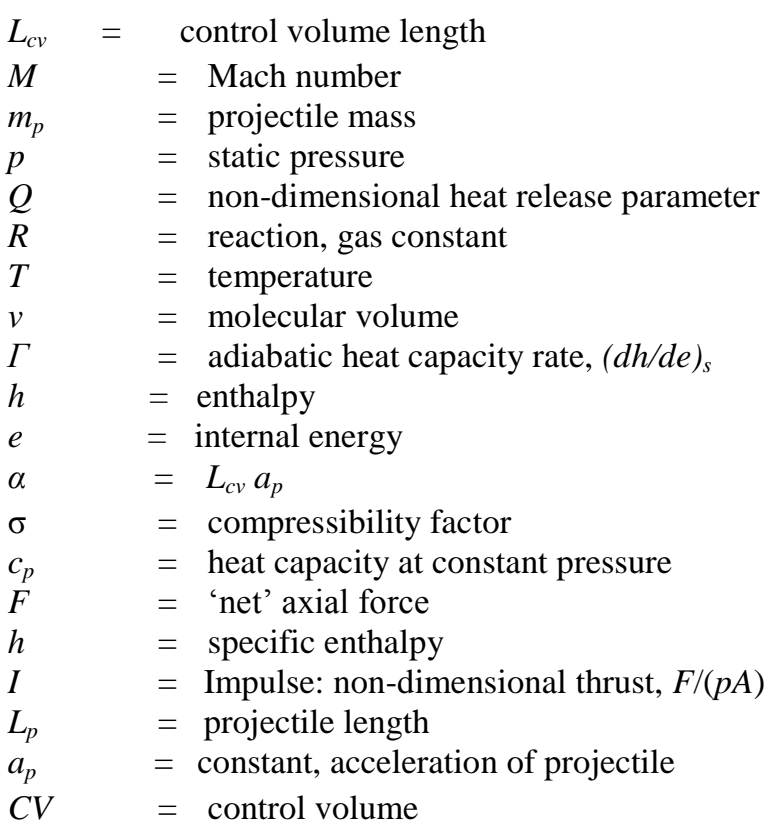

\section{Introduction}

The ram accelerator (Hertzberg et al. 1988), henceforth referred to, as RAMAC for brevity, is a propulsion concept based on using shock-induced combustion processes to accelerate projectiles up to very high velocity at a supersonic speed in a tube prefilled with a gaseous combustible mixture. Since this novel concept was first introduced in 1983, extensive experimental studies have been carried out at laboratories around the world, notably at the University of Washington (UW), Seattle, WA, USA, where the 38-mm-bore RAMAC facility has been used and operated at propellant fill pressures up to $20 \mathrm{MPa}$ (Hertzberg et al. 1991, Bruckner et al. 1991, Bundy et al. 2004); at the French-German Research Institute Saint Louis (ISL), France, with 30-mm-bore and 90$\mathrm{mm}$-bore at fill pressures up to $4.5 \mathrm{MPa}$ (Giraud et al. 1998); at the US Army Research Laboratory (ARL), Aberdeen, MD, USA, with 120-mm-bore and fill pressures of $8 \mathrm{MPa}$ (Nusca et al. 1991, Kruczynski 1993); and at Tohoku University, Sendai, Japan, with 25 -mm-bore and fill pressures of $6 \mathrm{MPa}$ (Sasoh et al. 1996, 1999).

Three RAMAC propulsive cycles are possible: the sub-detonative propulsion mode or thermally choked mode (see Fig. 1), in which the projectile velocity $V$ is less than the Chapman-Jouguet (CJ) detonation velocity, $D_{C J}$; the trans-detonative mode $\left(V=D_{C J}\right)$; and the super-detonative mode $\left(V>D_{C J}\right)$. The RAMAC technology is a multi-discipline investigation domain where several types of expertise are required, including aerodynamics, chemistry, thermodynamics, and material behavior. The performance of such a device is also dependent upon its physical configuration, i.e. RAMAC tube diameter and length, projectile dimensions, cross-section shapes, and the length-to-diameter ratio $(L / D)$. In the sub-detonative mode, the thrust is generated by the high projectile base pressure resulting from a normal shock system that is stabilized on the body by thermal choking of the flow at the full tube area behind the projectile as shown in Fig. 1 (Hertzberg et al. 1991).

Numerical simulation of flow around RAMAC with chemical reaction (combustion) presents considerable difficulties (or just say 'is challenging'). Some of the largest uncertainties in the modeling of reacting flow are the chemical reaction rates and the coupling between thermo-chemical phenomena. The uncertainties about the thermo-chemical processes render calculations doubtful. Additionally, the shockwave/boundary-layer interference flow field between the projectile and the launch tube as well as the projectile wake can induce a region of recirculation flow. Modeling these regions can be critical to overall flow field solution quality. Both laminar and turbulent flow must be investigated. These aspects have been addressed in previous studies of 2-D axisymmetrical and 3-D numerical modelling (Bengherbia et al. 2006, 2009). While those analysis were not aimed at providing data about the type of existing interactions in the launch tube or their influence on the thrust, both 2-D and 3-D simulation, despite their complexity, can provide this type of additional information. The purpose of the present work is to present a 1-D modelling approach that can simplify the analysis of these complex phenomena.

Theoretical 1-D calculations have been successfully used to predict the thrust in the subdetonative propulsive mode (Bruckner et al. 1991). This 1-D model, which includes projectile acceleration effects, has been further extended to include real-gas equations of state (EoS) in order to account for the compressibility effects of the combustion products (Bruckner et al. 1991, Bauer et al. 1998, 2005, Bengherbia et al. 2010). The net thrust is determined after solving a set of conservation equations by means of an iterative procedure. Extensive modelling studies using computational fluid dynamics (CFD) have also been performed (Bengherbia et al. 2006, 2009) to simulate the reacting flow field in the RAMAC. 


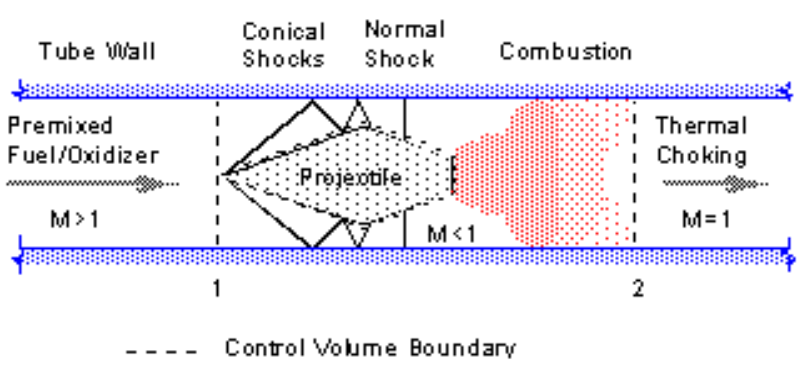

Figure 1. Flow over ram accelerator in the thermally choked propulsive mode.

A recent 1-D study using a performance code for the sub-detonative propulsion mode named TARAM was based on data derived from numerical simulations using a 3-D CFD code ANSYS-CFX (Bengherbia et al. 2011). The peak amplitude of pressure/temperature and the location of the shock wave system provided by CFD simulations were validated against available test data. In support of the 1-D modelling, an updated version of the computer code, TARAM (Bengherbia et al. 2010), was used which can calculate the thrust of the RAMAC thermally choked propulsive mode in both quasi-steady and unsteady conditions. It can also calculate the CJ detonation characteristics of the propellant mixtures. The results of these calculations were in good agreement with numerous experimental data obtained at the UW (USA) over a wide range of fill pressures; i.e., from $5 \mathrm{MPa}$ (quasi-steady modelling) up to $20 \mathrm{MPa}$ (unsteady modelling).

Computing the compressibility factor for a given EoS is the basis for incorporating the real-gas corrections. In order to calculate the characteristics of combustion products at elevated pressure, numerous equations of state based on generalized empirical and theoretical considerations have been applied (Bauer et al. 1985, Heuzé 1986). These formulations were also incorporated into the TARAM computer code, for which the appropriate choice of EoS is a key factor. The objective of this paper is to provide a comprehensive assessment of the best available formulations in order to improve the unsteady 1-D modelling and thus make the TARAM code a useful tool to predict the performance of the RAMAC in the sub-detonative velocity regime, without having to resort to more complicated 2-D or 3-D computational schemes.

The results of the computations are to be compared with representative test data from the UW 38-mm-bore and the ISL 90-mm-bore facilities. Among the numerous series of available data, the UW 38-mmbore results used for comparison with the predictions presented here are from a 16-m-long tube filled with a $2.95 \mathrm{CH}_{4}+2 \mathrm{O}_{2}+5.7 \mathrm{~N}_{2}$ propellant at a pressure of
5.0 MPa using a titanium alloy projectile having mass of $109 \mathrm{~g}$. In this experiment, the projectile entered the test section at $1030 \mathrm{~m} / \mathrm{s}$ and accelerated throughout its length to an exit velocity over $2000 \mathrm{~m} / \mathrm{s}$. The ISL 90$\mathrm{mm}$-bore results were from a $18-\mathrm{m}$-long tube filled with a $3.25 \mathrm{CH}_{4}+2 \mathrm{O}_{2}+9.8 \mathrm{~N}_{2}$ mixture at a pressure of 4 $\mathrm{MPa}$. A four-fin aluminium projectile with a mass of $1332 \mathrm{~g}$ was used (Giraud et al. 1998). These test data at two different size scales while using similar projectile geometries, propellants, fill pressures, and velocity range will be used to explore the efficacy of the unsteady, real-gas 1-D model presented here.

\section{One-dimensional model}

The one-dimensional, sub-detonative RAMAC model was originally developed at the University of Washington (Hertzberg et al. 1988, 1991). In this model (Figure 2), steady flow is assumed to enter the control volume at supersonic velocity (denoted as state 1) and to exit at sonic velocity (denoted as state 2) where it has attained chemical equilibrium while conserving mass and energy. The stream momentum thrust difference and the pressure differential between the incoming and outgoing flows is the thrust applied to the projectile. The predicted thrust of this thermally choked propulsive mode model compares very well with experiments when the rate of acceleration and the fill pressure are below $10,000 \mathrm{~g}$ and $2 \mathrm{MPa}$ respectively (Bruckner et al. 1991, Bundy et al. 2004).

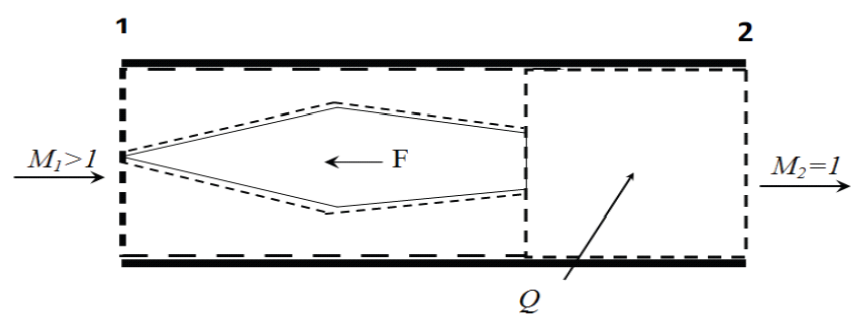

Figure 2. One-dimensional model of the RAMAC thermally choked propulsive mode.

At a higher acceleration level and elevated initial pressure, an investigation on the EoS of the unreacted mixture was undertaken (Redlich et al. 1949, Bauer et al. 2003). Moreover, a revised unsteady model that includes the effects of a real-gas EoS for the combustion products was developed (Bundy et al. 2004, Bauer et al. 2005). This model determines the effect of projectile acceleration on the net thrust as a global process between the state of the propellant entering the control volume and the state of the thermally choked exit flow (Bundy et al. 2004, Bauer et al. 2005). In the reference frame of the projectile, the mass, energy, and momentum conservation equations are applied to the propellant flow entering 
and leaving the control volume, which has a length $L_{C V}$. Conditions at the entrance and exit planes in Fig. 2 are identified by subscripts "1" and "2," respectively. The influence of combustion heat release on the rate of change of axial stream thrust is determined by the introduction of a non-dimensional chemical heat release parameter, $Q=\Delta q / C_{p l} T_{l}$ (where $\Delta q$ is the propellant heat release per unit mass, $c_{p}$ is constant pressure specific heat, and $T$ is static temperature) and net axial force of the projectile acting on the control volume, $F$, which is equal to the predicted RAMAC thrust.

Analysis of all the terms in these equations yields a readily applicable set of equations in the form expressed by Bundy et al. (Bundy et al. 2004, Bauer et al. 2005). After some algebraic manipulation of these relationships, while specifying the end state to be thermally choked, i.e., $M_{2}^{2}=\Gamma_{2} R_{2} T_{2}=1$, and introducing a real-gas EoS, namely, $p v / R T=\sigma(v, T)$, the following expressions are derived:

$$
\frac{T_{2}}{T_{1}}=\frac{c_{p 1}\left(\eta_{1}+\frac{\left(\Gamma_{1}-1\right) M_{1}^{2}}{2}+Q\right)}{c_{p 2}\left(\eta_{2}+\frac{\Gamma_{2} R_{2} M_{2}^{2}}{2 c_{p 2}}\right)}
$$

$$
\begin{array}{r}
P=\frac{M_{1} \sigma_{2} \sqrt{\Gamma_{1} R_{2}}}{M_{2} \sigma_{1} \sqrt{\Gamma_{2} R_{1}}} \sqrt{\frac{c_{p 1}\left(\eta_{1}+\frac{\left(\Gamma_{1}-1\right) M_{1}^{2}}{2}+Q\right)}{c_{p 2}\left(\eta_{2}+\frac{\Gamma_{2} R_{2} M_{2}^{2}}{2 c_{p 2}}\right)}} \\
I=\frac{M_{1} \sigma_{2} \sqrt{\Gamma_{1} R_{2}}}{M_{2} \sigma_{1} \sqrt{\Gamma_{2} R_{1}}} \sqrt{\frac{c_{p 1}\left(\eta_{1}+\frac{\left(\Gamma_{1}-1\right) M_{1}^{2}}{2}+Q\right)}{c_{p 2}\left(\eta_{2}+\frac{\Gamma_{2} R_{2} M_{2}^{2}}{2 c_{p 2}}\right)}} \\
\quad \times\left(1+\frac{\Gamma_{2} M_{2}^{2}}{\sigma_{2}}\right)-\left(1+\frac{\Gamma_{1} M_{1}^{2}}{\sigma_{1}}\right)
\end{array}
$$

with:

$$
\left.\alpha=L_{C V} a_{p} \quad ; \quad \Gamma=\frac{\partial h}{\partial e}\right)_{S}
$$

and:

$$
I=\frac{F}{p_{1} A_{1}} \quad ; \quad P=\frac{p_{2}}{p_{l}}
$$

$\sigma_{1}$ and $\sigma_{2}$ are the values of the compressibility factor in the fresh mixture and burned gases, respectively, $R$ is the gas constant, $\eta$ is the ratio of sensible enthalpy to the product of constant pressure specific heat and static temperature of the unreacted propellant, and $\gamma$ is the specific heat ratio of unreacted propellant.
Unlike in the quasi-steady-state assumption, the preceding equations show that the non-dimensional thrust, i.e., impulse $I$, is a direct function of both the length of the control volume, $L_{C V}$, and the projectile acceleration, $a_{p}$. An iterative procedure was used to solve for the value of $\alpha$ in Eq. (3) for an arbitrarily chosen value for $L_{C V}$ (Bauer et al. 2005). Based on experimental observations of the luminosity of the flow in 38-mm-bore experiments, a value of $L_{C V}=2 L_{P}$ was chosen (306 $\mathrm{mm}$ in this instance). Nevertheless, in order to refine this assumption, CFD calculations were used to investigate the variation of the control volume length at various incoming flow velocities (i.e., projectile velocities). The CFD determined control volume length dependence on velocity (and thus Mach) was based on the average axial coordinate of sonic contour in the combustion zone behind the projectile.

Importing the velocity-dependent CFD data of the control volume length into the 1-D unsteady analytical model provided a much better agreement with experimental data than the quasi-steady model using the ideal gas EoS (Bengherbia et al. 2011, 2012) (Figure 3).

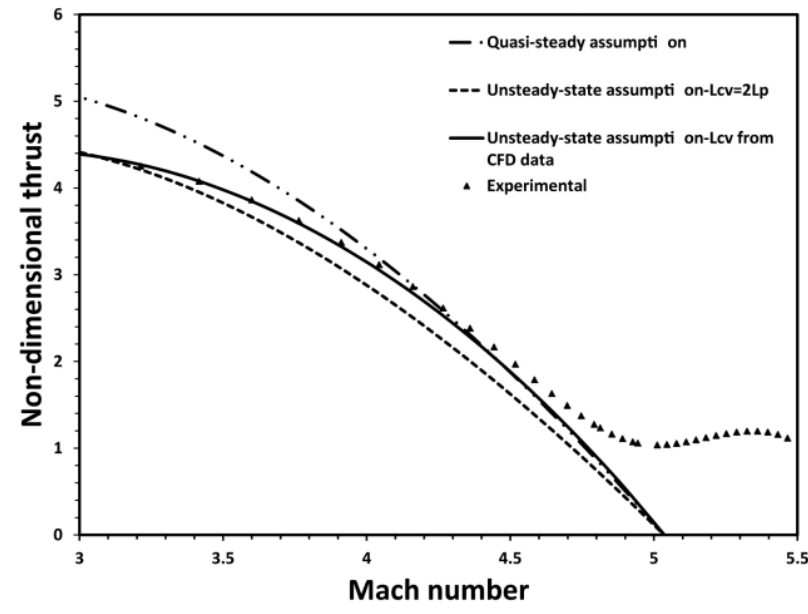

Figure 3. Improvement of the 1-D model of the RAMAC thermally choked propulsive mode based on ideal gas EoS and CFD data.

The calculated non-dimensional thrust based on the unsteady calculation is less than that derived from the quasi-steady assumption at all sub-detonative Mach numbers, whichever EoS is chosen. This difference is attributed to the increase of mass of the propellant accumulating in the control volume, such that it approaches the mass of the projectile itself (Bundy et al. 2004). It becomes significant when the projectile is experiencing very high accelerations; i.e., greater than 30,000 g (Bauer et al. 2005); however, it 
starts to be observable in the present case where the acceleration levels are moderate (i.e., $5000-20000$ g).

A propulsive mode transition occurs when the projectile reaches approximately $90 \%$ of the CJ speed. Since the current calculation predict the RAMAC performance when the flow behind the projectile is thermally choked; i.e., where the condition is $M_{2}=1$ at station 2 , it ceases to be reliable as the projectile velocity approaches the propellant $\mathrm{CJ}$ speed and the combustion moves up onto the projectile body. Experimental results deviate from theory at around $\mathrm{M}=4.6$, which is indicative of the onset of the RAMAC propulsive mode that operates in the transdetonative velocity regime. A complete discussion on this aspect is provided by Bengherbia et al. (Bengherbia et al. 2012) and Bruckner et al. (Bruckner et al. 1991).

The influence of real-gas effects does not account for discrepancy between theory and experiment at Mach numbers less than 4 . It has been observed in many experiments, however, that the projectiles erode and potentially contribute energy via metal combustion after $\sim 5$ milliseconds of operation; thus it is possible that these effects are playing a significant role here. Incorporating solid combustion modelling may be appropriate for these experiments (Devito et al. 2013).

\section{Available equation of state $(\mathrm{EoS})$ of the products}

Even though the agreement between 1-D modelling and experiment is very good for results shown in Fig. 3 when using the CFD-determined control volume length and assuming ideal gas EOS, it is of interest to develop the ability to incorporate EoS's that are better appropriate for RAMAC operation at fill pressures in the range of 7 to $30 \mathrm{Mpa}$.

Computing the compressibility factor for a given EoS is the basis for incorporating real gas corrections. Numerous equations of state have been developed based on generalized empirical and theoretical considerations were provided by Heuzé (Heuzé 1986). Only the general forms of each EoS incorporated in TARAM are presented here.

At this point several equations of state are correctly suited to predict the thermo-chemical properties of combustion products. Depending on the pressure range some are more pertinent to use. In the present case, a virial type, namely the Boltzmann EoS has been extensively used and its applicability to the RAMAC calculations has been widely demonstrated. However, the use of another EoS based on adjustable parameters that could be suited to fit the present use is worth being investigated. For this purpose, the Becker Kistiakowsky and Wilson (BKW) EoS is investigated here. The main reason is its applicability to a wide range of temperatures and pressures of combustion products that cover the whole field of gaseous to condensed explosives, based on the appropriate choice of the adjustable parameters.

\subsection{Boltzmann}

The Boltzmann EoS (Bauer et al. 1981) adequately predicts the Chapman-Jouguet properties when the pressure of combustion products does not exceed 200 MPa (Bauer et al. 1985). This equation of state treats the individual molecules as hard spheres and the mixing rule only accounts for interactions of similar species. The Boltzmann expansion for the compressibility factor is computed by the formula:

$\sigma=1+x+0.626 x^{2}+0.287 x^{3}+0.193 x^{4}$

where $\mathrm{x}$ is defined as:

$$
x=\sum_{i} \frac{X_{i} B_{i}}{v_{i}}
$$

where $B_{i}$ is the covolume, $X_{i}$ is the mole fraction and $v_{i}$ is the specific volume of species $i$ (Heuzé 1986).

\subsection{Becker-Kistiakowsky-Wilson (BKW)}

This EoS was introduced in 1921 by Becker, and later modified by Kistiakowsky and Wilson (Mader 1963). It can be presented as follows:

$$
\frac{p v}{R T}=1+x \times e^{(\beta x)}
$$

with:

$$
x=\frac{\kappa B}{V(T+\theta)^{\alpha}}
$$

and:

$$
B=\sum_{i} x_{i} B_{i}
$$

where $\alpha, \theta, \kappa, B_{i}$ are semi-empirical constants that must be adjusted. In particular, $B_{i}$ are the co-volumes and there is no link to the co-volume defined by Boltzmann equation of state. This form of EoS is mostly used for condensed explosives; however, previous research by Heuzé (Heuzé 1986) and 
Bengherbia et al. (Bengherbia et al. 2010) showed that it could be used for the calculation of gaseous detonation characteristics at extremely elevated pressure. In which case, all the adjustable parameters must be set accordingly.

\section{Results and discussion}

Among a series of EoS, the 1-D modelling of TARAM code is capable of using the ideal gas, Boltzmann, or BKW EoS; which were validated by comparison with experimental CJ speeds (Bauer et al. 1991, 1996). At this time, most studies on RAMAC thrust and velocity profiles have used the Boltzmann EoS for the calculation of combustion products and the ideal gas EoS for the reactants. In the specific case, regardless the propellant mixture, TARAM is able to calculate the real gas effects for each EoS by changing the way $\sigma$ is computed.

In order to investigate the applicability of these EoS for predicting thrust of the RAMAC thermally choked propulsive mode, the present study is aimed at comparing both the calculated velocity profiles and non-dimensional thrust with experimental results.

\subsection{Velocity-distance}

The experimental data from the UW are plotted in Fig. 4 along with the 1-D modelling results using the Boltzmann and the BKW EoS and the CFD determined control volume of velocity dependence. It is evident that the Boltzmann predictions agree very well up to a velocity of $\sim 1750 \mathrm{~m} / \mathrm{s}$, which is $\sim 0.95 D_{C J}$ predicted by this EoS. Whereas the BKW results over predict performance for this situation. This latter result is not unexpected since the CJ speed predicted for this propellant by BKW EoS is about $10 \%$ higher than that measured in experiments at this fill pressure.

The ISL experimental data are plotted in Fig. 5 along with the 1-D modelling results using the Boltzmann and the BKW EoS and the CFD determined control volume dependence on velocity. Note that in this case the control volume length was geometrically scaled from CFD results for $38-\mathrm{mm}$ bore and the ratio of propellant-to-projectile density ratio was over an order of magnitude less for the 90$\mathrm{mm}$ experiment compared to that of the $38-\mathrm{mm}$ experiment. The low propellant-to-projectile density ratio is consistent with the acceleration level being about $1 / 5^{\text {th }}$ that of the $38-\mathrm{mm}$-bore experiment. In this case the BKW predictions agreed the experiment better than those based on the Boltzmann EoS.

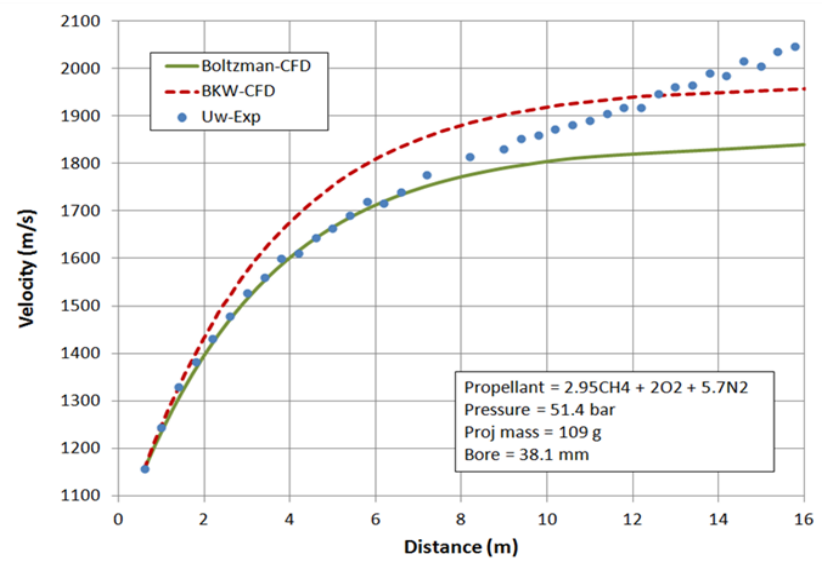

Figure 4. Experimental and theoretical velocity-distance

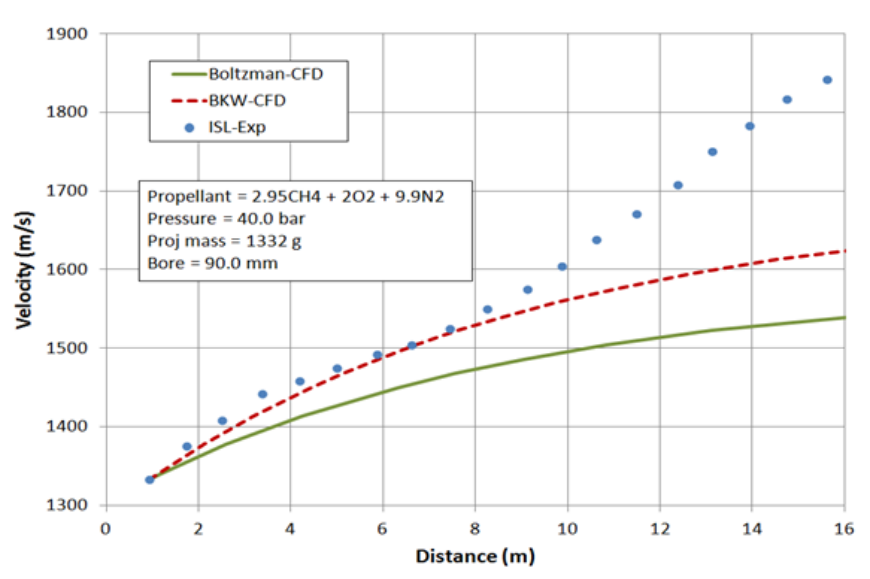

Figure 5. Experimental and theoretical velocity-distance

\subsection{Non-dimensional thrust}

The experimental data from the UW (38-mmbore) are plotted along with the theoretical results using the ideal gas (Fig. 6), Boltzmann (Fig. 7), and BKW (Fig. 8) EoS and the CFD determined control volume dependence on velocity. As previously demonstrated, the ideal gas EoS underpredicts the thrust in the region near the $\mathrm{CJ}$ detonation speed (Fig 3). It is evident that the Boltzmann EoS modeled the thrust behavior within 3\% over the Mach range of 3.2 to 4.6 , which is $\sim 0.95 D_{C J}$ predicted by these EoS. Here again, the BKW results over predict performance for this situation, as observed with the velocity data. 


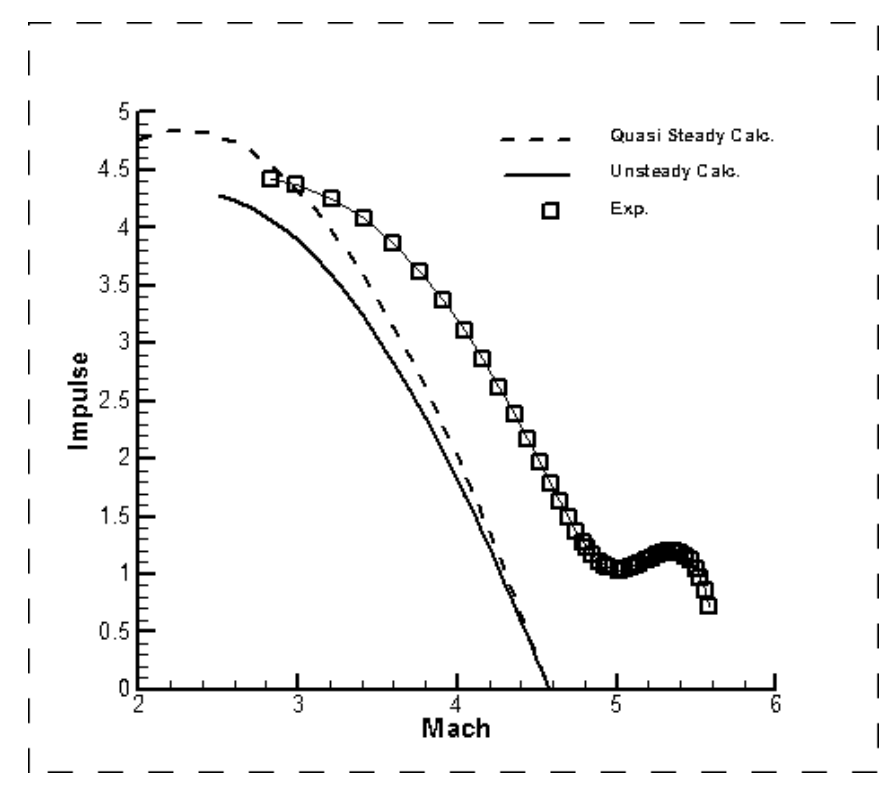

Figure 6. Non-dimensional Thrust-Mach-Number plot for $2.95 \mathrm{CH}_{4}+2 \mathrm{O}_{2}+5.7 \mathrm{~N}_{2}$ propellant, $p_{0}=5.0 \mathrm{MPa}$ (Ideal gas EoS was used both at station 1 and for the calculation of properties of combustion products)

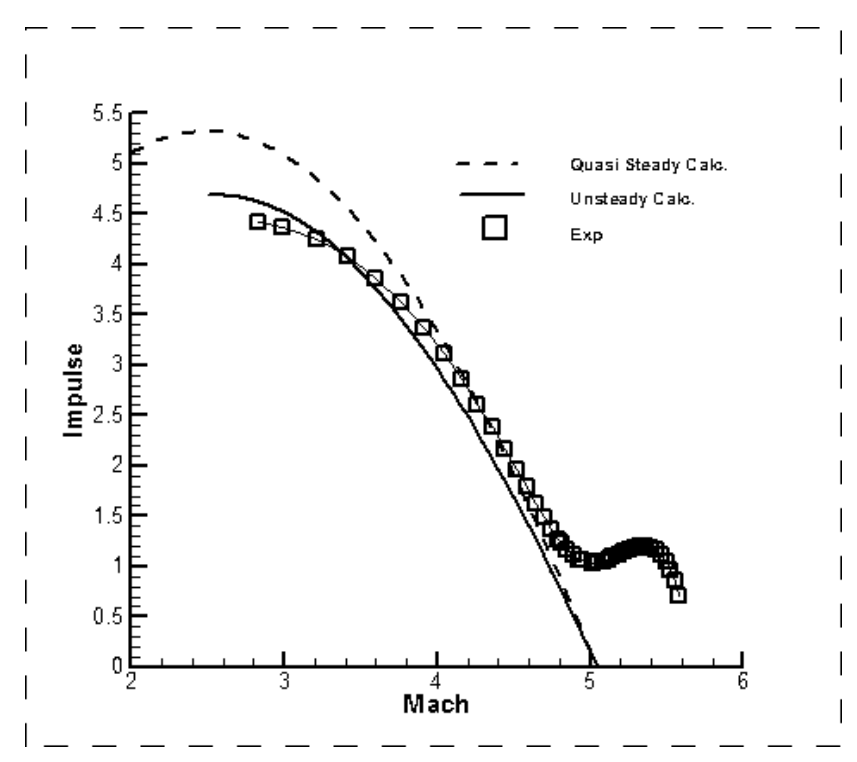

Figure 7. Non-dimensional Thrust-Mach-number plot for $2.95 \mathrm{CH}_{4}+2 \mathrm{O}_{2}+5.7 \mathrm{~N}_{2}$ propellant, $p_{0}=5.0 \mathrm{MPa}$ (Ideal gas EoS was used at station 1 and Boltzmann EoS was used for the calculation of properties of combustion products)

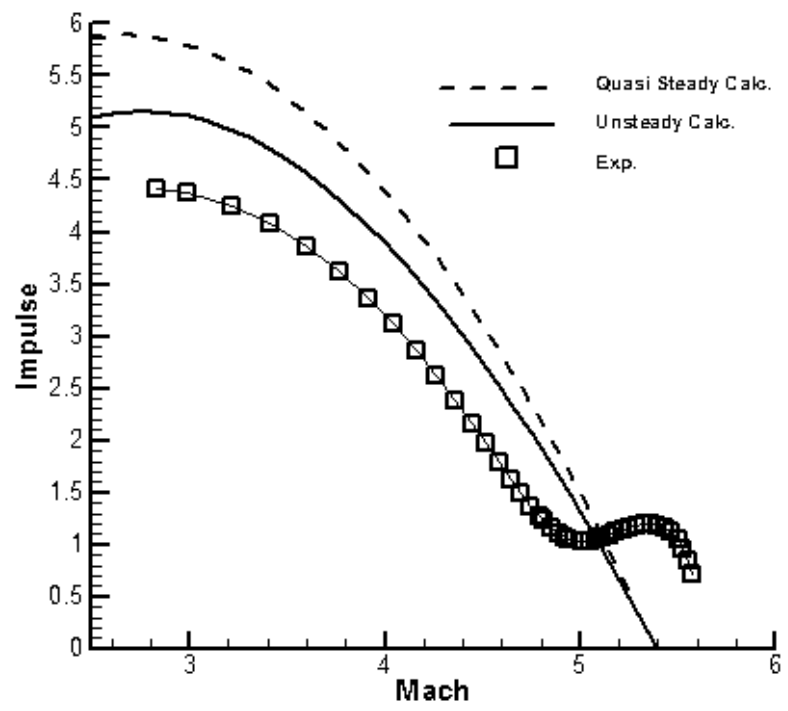

Figure 8. Non-dimensional Thrust-Mach-number $\overline{\text { plot }} \overline{\text { for }}$ $2.95 \mathrm{CH}_{4}+2 \mathrm{O}_{2}+5.7 \mathrm{~N}_{2}$ propellant, $\mathrm{p}_{0}=5.0 \mathrm{MPa}$ (Ideal gas EoS was used at station 1 and BKW EoS was used for the calculation of properties of combustion products)

The ISL experimental data (90-mm-bore) are plotted in Fig. 9 along with the theoretical results using the Boltzmann and the BKW EoS and the CFD determined control volume dependence on velocity. The discrepancy in the thrust-velocity predictions of the TARAM code when using Boltzmann and BKW EoS for 38-mm-bore and 90-mm-bore may be due to the control volume length scaling used in these computations, this matter will be investigated in more detail with large-scale CFD modelling. It may improve the slight disagreement that remains in the lower range of Mach numbers.

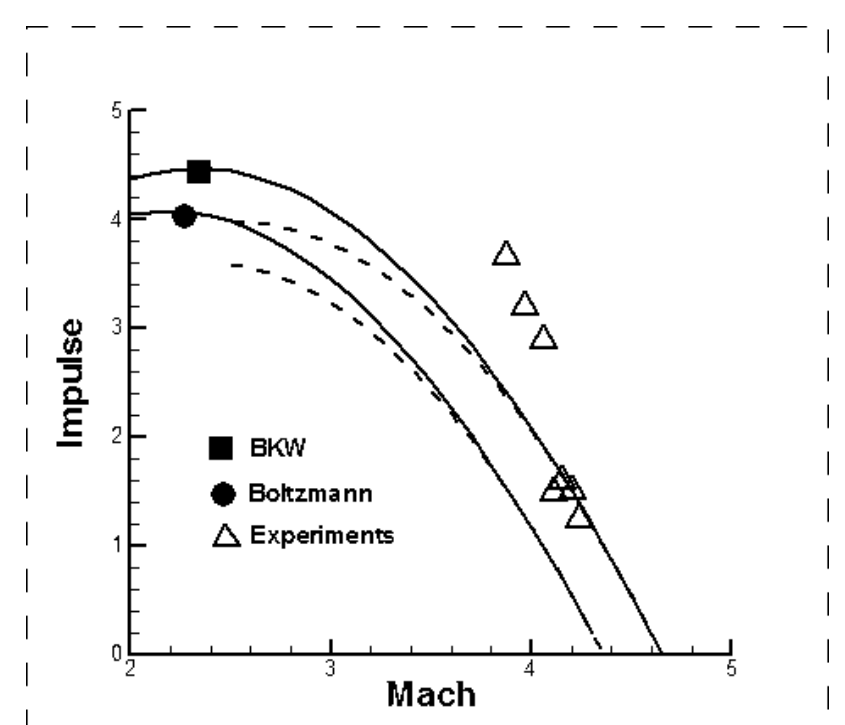

Figure 9. Non-dimensional Thrust-Mach-number plot for $2.95 \mathrm{CH}_{4}+2 \mathrm{O}_{2}+9.9 \mathrm{~N}_{2}$ propellant, $\mathrm{p}_{0}=4.0 \mathrm{MPa}$ (Ideal gas EoS was used at station 1 and Boltzmann and BKW EoS's 
were used for the calculation of properties of combustion products; solid and dashed lines are quasi-steady and unsteady assumption, respectively).

\section{Summary and conclusion}

A computer program TARAM, which is a onedimensional code, calculates the RAMAC characteristics on the basis of quasi-steady and unsteady assumptions, including two real-gas equations of state and an ideal gas option. The additional data provided by 3-D CFD simulation in terms of control volume length made TARAM even more reliable as a prediction tool of RAMAC performance. This is specifically the case when the CFD data have been validated on the same facility as that used for the calculations. A comparison of the computed results with experiments shows that, among all of the equations of state considered, the Boltzmann model, despite its simple molecular interaction law, is appropriate for $5 \mathrm{MPa}$ operating conditions at 38- $\mathrm{mm}$ bore. Although it tends to overpredict the experimental data from the 38-mm-bore tube test, the empirical BKW EoS turns out to improve the theoretical agreement with experimental data from the $90-\mathrm{mm}$ bore tube test. The calculation based on the unsteady model is generally in better agreement with experiments than those using a quasi-steady model but it does not exhibit a major shift of the values, at least at the initial pressures investigated (e.g. 4 to $5 \mathrm{MPa}$ ). The main question that might rise is whether the differences observed in the comparison graphs between 1-D modelling and experiment can be due to some physical phenomena that have been neglected in the 1-D model. However, since the same assumptions were used for each EoS; i.e., same real-gas corrections and analytical procedure, it can be stated that these differences can solely be attributed to the equation of state itself rather than code implementation.

\section{REFERENCES}

Bauer, P., Brochet, C., Krishnan S (1981), 'Detonation Characteristics of Gaseous Ethylene, Oxygen and Nitrogen Mixtures at High Initial Pressures', Progress in Aeronautics and Astronautics, Ed. AIAA, New York, NY, Vol. 75, pp. 408-422.

Bauer, P., Presles, H.N., Heuzé, O., Brochet, C. (1985), 'Equation of State for Dense Gases', Archivum Combustionis, 5, 1, pp. 315-320.
Bauer, P., Dunand, M. and Presles, H.N. (1991) 'Detonation characteristics of gaseous methaneoxygen-nitrogen mixtures at extremely elevated initial pressure', AIAA, Progress in Aeronautics and Astronautics, Vol. 133, pp. 56-62.

Bauer, P., Legendre, J.F., Knowlen, C., and Higgins, A.J. (1996) 'Transition of Detonation on Insensitive Dense Gaseous Mixtures in Tubes', AIAA 96-2682.

Bauer, P., Knowlen, C., Bruckner, A.P. (1998) 'Real gas effects on the prediction of ram accelerator performance', Shock Waves, Vol. 8, pp. 113118.

Bauer, P., Knowlen, C. (2003) 'Compressibility effects of unreacted propellant on thermally choked ram accelerator performance', Eur. Phys. J. Appl. Phys., Vol. 21, pp.233-238.

Bauer, P., Knowlen, C., Bruckner, A.P. (2005) 'Modeling Acceleration effects on Ram Accelerator Thrust at High Pressures', AIAA J. of Propulsion and Power, Vol. 21, pp. 955-95

Bengherbia, T., Yao, Y.F., Bauer, P. (2006) 'Computational Investigation of Transitional Viscous Flow over a Ram Accelerator Projectile in Sub-Detonative Propulsion Mode', AIAA 2006-0558.

Bengherbia, T., Yao Y., Bauer P., Knowlen C. (2010) 'One-dimensional Performance Modeling of the RAMAC in Subdetonative Regime', Aerotecnica, J. of Aerospace Sci., Tech. and Syst., Vol. 89, pp. 3-13.

Bengherbia, T., Yao Y., Bauer P., Giraud M., Knowlen C. (2011), 'Improved 1-D unsteady modeling of the thermally choked RAMAC in the sub-detonative propulsion mode', J. of Appl. Mech., Vol. 78, pp. 150-167.

Bengherbia, T., Yao Y., Bauer P., Knowlen C. (2012), 'CFD-based 1D Modeling of the Thermally Choked Ram Accelerator', the 50 AIAA Aerospace Sciences Meeting and Exhibit, Nashville, TN, AIAA 2012-982

Bengherbia, T., Yao, Y.F., and Bauer, P., Knowlen, C. (2009) 'Numerical Investigation of Thermally Choked Ram Accelerator in Sub-Detonative Regime', AIAA 2009-0635.

Bruckner, A.P., Knowlen, C., Hertzberg, A., and Bogdanoff, D.W. (1991) 'Operational Characteristics of the Thermally Choked Ram Accelerator', Journal of Propulsion and Power, Vol. 7, No. 5, pp.828-836.

Bundy, C., Knowlen, C., Bruckner, A.P. (2004) 'Unsteady Effects on Ram Accelerator Operation at Elevated Fill Pressures', Journal of Propulsion and Power, Vol. 20, pp. 801-810. 
Devito, A.M., Higgins A. (2013) 'Ignition Envelope for Supersonic Combustion of Bulk Metals', the $51^{\text {st }}$ AIAA Aerospace Sciences Meeting and Exhibit, Grapevine, TX, AIAA 2013-0888.

Giraud, M. J., Legendre, J.F., and Henner, M. (1998) 'RAMAC in Subdetonative Propulsion Mode: State of the ISL Studies', Ram Accelerators, Takayama K., Sasoh A. (eds), Springer-Verlag, Heidelberg, pp. 65-78.

Hertzberg, A., Bruckner, A.P., and Bogdanoff, D.W. (1988) 'Ram Accelerator: A New Chemical Method for Accelerating Projectiles to Ultrahigh Velocities', AIAA Journal, Vol. 26, No. 2, pp. 195-203.

Hertzberg, A., Bruckner, A.P., and Knowlen, C. (1991) 'Experimental Investigation of Ram Accelerator Propulsion Modes', Shock Waves, Vol. 1, No. 1, pp. 17-25.

Heuzé, O., (1986) 'Equations of State of Detonation Product', Phys.Rev. A, Vol. 34, pp. 428-432.

Knowlen, C., Higgins, A.J., Bruckner, A.P., Bauer, P. (1996) 'Ram accelerator operation in the superdetonative velocity regime', AIAA 960098.

Kruczynski, D.L. (1993) 'New experiments in a 120mm ram accelerator at high pressures', the $29^{\text {th }}$ AIAA, SAE, ASME, and ASEE Joint Propulsion Conference and Exhibit, Monterey, CA.

Nusca M.J., Kruczynski, D.L (1991) 'Reacting Flow Simulation for Large-Scale RAMAC', Journal of Propulsion and Power, Vol. 12, No. 1, pp. 61-69.

Mader, C.L. (1963) 'Detonation Properties of Condensed Explosives Computed Using the Becker-Kistiakosky-Wilson Equation of State', Report-LA-2900, Los Alamos Scientific Laboratory, NM, USA.

Redlich, O. and Kwong, J.N.S. (1949) 'On the Thermodynamics of Solutions. V: An Equation of State. Fugacities of Gaseous Solutions', Chem. Rev., Vol. 44(2), pp. 233-244.

Sasoh, A., and Hamate, Y., Utsunomiya, G. and Takayama, K. (1999) 'High acceleration ram accelerator operation', the $35^{\text {th }} A I A A, S A E$, ASME, and ASEE Joint Propulsion Conference and Exhibit, Los Angeles, CA.

Sasoh, A., Higgins, A.J., Knowlen, C., and Bruckner, A.P. (1996) 'Hollow projectile operation in the RAMAC', J. Propulsion and Power, Vol. 12, pp. 1183-1186. 